\title{
Sitzung vom 12. December 1881.
}

\author{
Vorsitzender: Hr. A. W. H ofmann, Vice-Präsident.
}

Das Protocoll der letzten Sitzung wird genehmigt.

Der Vorsitzende beklagt, dass ihm die traurige Pflicht obliege, der Gesellschaft einen schmerzlichen Verlust anzuzeigen.

Prof. Wilb. Weith, seit einer Reibe von Jahren eines der thätigsten Mitglieder unserer Gesellschaft, ist am 29. November in der Blüthe der Jahre der Wissenschaft und seinen Freunden entrissen worden. Derjenige, der Ihnen diese Trauerkunde meldet, hat nicht Gelegenheit gehabt, die persönliche Bekanntschaft des Verewigten zu machen, er ist daher auch nicht in der Lage, Ibnen mehr als einige ganz dürftige Angaben über seinen Lebens- und Bildungegang mitzutheilen. Aber schon hält eine befreundete Hand die Feder, welche berufen ist, das Lebensbild des Geschiedenen za zeichnen.

Prof. Weith war am 9. Mai 1846 in Homburg vor der Höh' geboren. Er studirte in Heidelberg und Zürich. An der letztgenannten Universität habilitirte er sich gegen Ende der sechziger Jahre, wurde 1871 zum ausserordentlichen und 1874 zum ordentlichen Professor ernannt. Gleichzeitig bekleidete er eine Professur an dem eidgenössischen Polytechnicum in Zürich. In diesen Stellungen hat Weith die Reihe schöner Arbeiten ausgeführt, durch welche ihn die Mitglieder unserer Gesellschaft, der er fast von ibrer Bildung an angehört hat, als unermüdlichen und erfolgreichen Forscher auf dem Felde der organischen Chemie kennen und schätzen gelernt haben. Es kann am heatigen Abend meine Aufgabe nicht sein, eine Skizze der umfassenden Lebensarbeit des so früh Dahingeschiedenen auch nur zu versuchen. Gleichwobl möchte ich nicbt unterlassen, wenigstens an einige seiner wichtigsten Forschungen zu erinnern.

Es waren zunlichst mehrfache Beobachtungen über Cyankörper, namentlich eingehende Versuche mit den Nitroprussidverbindangen, dorch welche sich der junge Forscher bei den Chemikern einführte.

Berichte d. D. chem. Gesellschaft. Jabrg. XIV. 
Diesen folgte bald eine erfolgreiche Bearbeitung zahlreicher Glieder der aromatischen Reihe. Unter den Reactionen, die Weith mit Vorliebe studirte, muss die Umwandlung der Isonitrile in Senföle und die Rückbildung der ersteren aus letzteren genannt werden, sowie die Ueberführung der Isonitrile in die normalen Nitrile und die auf diese Weise vermittelte Möglichkeit des Aufsteigens von den aromatischen Monaminen zu den zugehörigen Säuren, ein Uebergang, welcher zur Lösung mannichfacher, damals schwebender Fragen der Atomstellung erwünschte Beiträge geliefert hat. Von den Senfölen zu den Harnstoffen und Guanidinen ist nur ein Scbritt, und so finden wir Weith auch bald mit umfassenden Untersuchungen über diese Körpergruppen beschäftigt. Die Entschwefelung des Diphenylsulfoharnstoffs bei Abwesenheit des Wassers, die Erkenntniss, dass der Bildung des Oxyharnstoffs die einer cyanamidartigen Verbindung, des Carbodjphenylimids, voransgebt, die Darstellang eines Tetraphenylguanidins aus Diphenylamin und Chlorcyan, and endlich die scharfsinnige Aufklärung der Constitution des Carbotriphenyltriamins durch die elegante Synthese dieses Körpers ans Anilin und Paranitrobenzoësäure sind einige wenige der zahlreichen Ergebnisse, zu denen diese Untersucbungen geführt baben. Weiter mag hier, wenn auch nur vorübergehend, der Umwandlung der Orthotoluylsäure und des Antbrachinons in Phtalsäare gedacht werden; endlich seiner Abhandlung über die Constitution der Guanamine, welche zur Klarlegung dieser Körpergruppe wesentlich beigetragen hat. Ebenso umfangreich und wichtig aber als die Forschungen, welche wir Weith allein verdanken, sind die Arbeiten, welche er gemeinschaftlich mit seinem Freande und Collegen Victor Merz ausgefübrt hat. Unter diesen sind die umfassenden Versuche über die Entschwefelung der Sulfoharnstoffe, welche unsere Kenntniss der Gruppe der Guanidine so sehr erweitert baben, über Darstellung und Trennung der Naphtalinsulfosäuren, über die Einwirkung des Schwefels auf Anilin und seine Homologen, welche zur Entdeckung der geschwefelten, aromatischen Amine gefübrt haben, über zahlreiche Abrömmlinge des Diphenylamins, über die Bildung des Tripbenylamins, über die erschöpfende Bromirang der Kohlenwasserstoffe in erster Linie zu nennen. Wie sehr den Freunden aber auch der didaktiscbe Ausbau unserer Wissenschaft am Herzen lag, baben sie durch die Beschreibung zahlreicher, interessanter Vorlesungsversuche betbätigt; es dürfte kaum ein Docent sein, der seinen Zubörern den Borsänreflammenversuch nicht in der einfachen und eleganten Anordnung zeigte, welche wir Weith und Merz verdanken.

$\mathrm{Zu}$ den letzten Aufgaben, welche sich der Verstorbene, gleichfalls in Gemeiuschaft mit dem überlebenden Freunde, stellte, gebörte die Einführung der Phenylgruppe und analoger Gruppen bei Gegenwart von Metallchloriden einerseits in Phenole, andererseits in Amine. 
So gelang endlich die langangestrebte Umwandlung des Ammoniaks in Anilin. Die zahlreichen interessanten Verbindungen, welche auf diese Weise gebildet werden, sind noch frisch in der Erinnernng der Mitglieder der Gesellschaft. Ist doch die letzte Abhandlung über diesen Gegenstand noch erst vor wenigen Wochen in unserer Mitte zur Verlesung gelangt.

Eine so unermãdliche, vielseitige Thätigkeit schien, neben bewundernswürdiger geistiger Regsamkeit, auch eine glücklich angelegte Körperconstitution zu bezengen. Gleichwobl war Prof. Weith schon seit einigen Jahren von einem Brastleiden heimgesucht, dessen Entwickelung seine Freunde in banger Sorge hielt. Mehr als einmal hatte sich der Leidende vor den Stürmen des Winters nach dem Süden geflüchtet. Aber er war stets im Frübjahr so weit gekräftigt zurückgekehrt, dass er sich von Neuem seinen Lieblingsstudien hiozugeben vermochte. Diesmal wollte er den Winter in Corsica zubringen. Aber schon bald nach der Landung ereilte ihn seine Stunde. Er starb am 29. November in Ajaccio. Mit ihm sind grosse Hoffnungen zu Grabe gegangen!

Friede seiner Asche, Ehre seinem Andenken!

Die Mitglieder der Gesellschaft erheben sich von ibren Sitzen.

Zu ausserordentlichen Mitgliedern werden proclamirt die Herren:

Hans Kreiss, Zürich;

Paul Näf, Rüschlikon bei Zürich;

Oscar Bielschowski,

L. v. Lesser,

R. Löwenthal,

Bernhard Dessau,

Alfred Hagen,

Fritz Eltzbacher,

Anton Schnell,

Henrich Bull, Basel;

Franklin C. Robingon, Brunswick, Maine.

Zu ausserordentlichen Mitgliedern werden vorgeschlagen die Herren:

A. E. Menke, Cambridge, Mass. U. S. A. (durch C. Ch. R. Sanger, Loring Jackson und H. B. Hill); F. A. A schmann, Sharon, Mercer Co. Penneylvanien (durcb V. Meyer und Edw. Keller); 
A. Behrmann, Berlin, Louisenplatz 1 (durcb S. Gabriel und P. Meyer);

P. Neymann, Berlin, Georgenstr. 35 (durcb S. Gabriel und J. Eisenberg);

Dr. E. Parcus, München, Arcisstr. 1 (durch E. Drechsel und H. Moeller);

Dr. G. Liebscher, Halle a./S. (durch G. Baumert und E. Schmidt);

Carl Müller, Braunschweig, Polytechnikum (durch L. Schulze und Fr. Salomon);

Heinrich Albert, Chem. Fabrik, Biebrich (durch W. Kalle und J. Wolff);

Dr. G. Prausnitz, Görlitz (durch Th. Schuchardt und F. Tiemanu);

Th. Bissinger, Erlangen (durch J. Volhard und A. H. Weigmann, Hilger);

E. Farnk op f, Riverside, Cincinnati O. (durch N. Delbrück und A. Schrohe);

E. Fürst, Prag, Chemische Fabrik von W. Brosche und Eisenberg (durch L. J. Eisenberg und F. Tiemann);

Dr. H. Pressler, Halle a./S. (durch E. Schmidt und G. Baumert);

F. Pfeifer, Graz (durch L. v. Pebal und A. W. Hofmann).

Für die Bibliothek sind als Geschenke eingegangen:

85. Naturen, Et illustreret Maanedsskrift etc. 1881. No. 11. (Voin Redactear.)

384. Kerl, Bruno. Metallurgische Probirkunst. 2. Aufl. Leipzig 1882. (Verf.)

385. Jünemann, Friedr. Die Fabrikation des Alauns der schwefelsauren und essigsauren Thonerde des Bleiweisses und des Bleizuckers. Wien, Pest, Leipzig 1882. (Verf.)

884. Korschelt, O. Japanischer Ackerboden, ein naturlicher Cement. Yokohama 1881. Sep,-Abdr. (Verf.)

1235. Pictet, Aimé. Recherches sur les éthers tartriques. Dissertation. Genève 1881. (Verf.)

1236. Classen, Alexunder. Quantitative Analyse auf electrolytischem Wege. Aachen 1882. (Verf.)

Der Schriftführer:

A. Pinner.
Der Vorsitzende:

A. W. $H$ of $m$ a n $n$. 